\title{
Compensating for ASD: masking the truth?
}

\section{By Dr. Jessica Edwards}

"Compensation" is a phenomenon by which individuals with Autism Spectrum Disorder (ASD) show improvements in overt symptoms, namely their understanding of others ("theory-of-mind", ToM), despite persisting deficits at the cognitive and neurobiological levels. Few studies, however, have investigated its mechanisms and impact on mental health. Now, data suggest that those demonstrating a high degree of compensation (i.e. have good social skills) have a better IQ and executive functioning but worse self-reported levels of anxiety compared to those demonstrating a low degree of compensation.

The study included 136 adolescents with ASD, who had been recruited to the ongoing Social Relationships Study. The participants were divided into two groups: (1) strong compensators, identified as those with good Autistic Diagnostic Observation Schedule (ADOS) scores despite poor ToM; and (2) poor compensators, being those with both poor ADOS scores and ToM. After completing a range of cognitive tasks and a self-reported anxiety questionnaire, the researchers identified that IQ, executive function and anxiety are involved in the process by which some adolescents with ASD compensate for social difficulties. Importantly, these "high compensators" are not experiencing a milder form of ASD than "low compensators". The researchers hope that these data will improve understanding of under-diagnosis and mental-health difficulties in ASD.

\section{References}

Livingston, L.A., Colvert, E., the Social Relationships Study Team, Bolton, P. \& Happé, F. (2018), Good social skills despite poor theory of mind: exploring compensation in autism spectrum disorder. $J$ Child Psychol Psychiatr, doi:10.1111/jcpp.12886 